\title{
Day 2 neutrophil-to-lymphocyte and platelet-to-lymphocyte ratios for prediction of delayed cerebral ischemia in subarachnoid hemorrhage
}

\author{
*William S. Bolton, MBChB,,2 Parjeet Kaur Gharial, MBBS, MRCS, ${ }^{2}$ \\ Christopher Akhunbay-Fudge, MRCS, ${ }^{2}$ Paul Chumas, MD, FRCS(SN), ${ }^{2}$ \\ Ryan K. Mathew, PhD, FRCS(SN), ${ }^{1,2}$ and lan A. Anderson, FRCS(SN) ${ }^{2}$ \\ ${ }^{1}$ Leeds Institute of Medical Research at St. James's, School of Medicine, University of Leeds; and 'Department of Neurosurgery, \\ Centre for Neurosciences, Leeds Teaching Hospitals NHS Trust, Leeds, United Kingdom
}

\begin{abstract}
OBJECTIVE Recent evidence has suggested that an admission neutrophil-to-lymphocyte ratio (NLR) of $\geq 5.9$ predicts delayed cerebral ischemia $(\mathrm{DCl})$ in aneurysmal subarachnoid hemorrhage $(\mathrm{aSAH})$. The primary aims of this study were to assess reproducibility and to ascertain the predictive ability of NLR on subsequent days postictus. Secondary aims included identification of additional inflammatory markers.

METHODS A single-center, retrospective study of all patients aged $\geq 18$ years with aSAH between May 2014 and July 2018 was performed. Patient characteristics, DCl incidence, operative features, and outcomes (on discharge and at 3 months postictus) were recorded. C-reactive protein (CRP) and full blood count differentials were recorded on admission and through day 8 postictus or at discharge. In total, 403 patients were included in the final analysis.

RESULTS Ninety-six patients (23.8\%) developed DCI with a median time from ictus of 6 days (IQR 3.25-8 days). A platelet-to-lymphocyte ratio (PLR) cutoff $\geq 157$ and CRP cutoff $\geq 27$ was used in our cohort. In a multiple binary logistic regression model, after controlling for known DCI predictors, day 2 NLR $\geq 5.9(\mathrm{OR} 2.194,95 \% \mathrm{Cl} 1.099-4.372 ; p=$ 0.026 ), day 1 PLR $\geq 157$ (OR 2.398, 95\% Cl 1.1072-5.361; $p=0.033$ ), day 2 PLR $\geq 157$ (OR 2.676, 95\% Cl 1.344$5.329 ; p=0.005)$, and $C R P \geq 27$ on days 3,4 , and 5 were predictive of $\mathrm{DCl}$.

CONCLUSIONS The results of this study have confirmed the association between NLR and DCl and have demonstrated the predictive potential of PLR and CRP, suggesting that NLR and PLR at day 2, and CRP from day 3 onward, may be better predictors of $\mathrm{DCl}$ than those measurements at the time of ictus.

https://thejns.org/doi/abs/10.3171/2021.12.FOCUS21642
\end{abstract}

KEYWORDS subarachnoid hemorrhage; biomarkers; blood cell count

$\mathrm{T}$ HERE are approximately 4800 cases of subarachnoid hemorrhage (SAH) in the United Kingdom each year, of which $70 \%$ are caused by a ruptured cerebral aneurysm. ${ }^{1} \mathrm{SAH}$ is a type of hemorrhagic stroke caused by bleeding in the subarachnoid space, and treatment often involves neurosurgical or endovascular intervention and medical stabilization. ${ }^{2}$ Approximately $15 \%$ of patients with SAH die before they reach the hospital, and for those who receive treatment, SAH carries a high morbidity risk and a 30-day mortality rate of $12.9 \% .^{1,2} \mathrm{~A}$ subset of patients with SAH experience a further neurological impairment in the form of delayed cerebral ischemia (DCI), which confers a poorer clinical prognosis. ${ }^{3}$
DCI is defined as a new neurological impairment within 6 weeks of SAH onset and is estimated to affect $30 \%$ to $40 \%$ of patients with SAH. ${ }^{3}$ The pathophysiology of DCI is poorly understood, and being able to predict those patients at greatest risk of developing DCI is an ongoing research priority. ${ }^{3}$

While identifying patients at greatest risk may allow prompt intervention before the onset of DCI and, thus, improve outcomes, predictive variables have been challenging to identify. ${ }^{4}$ Recently, Al-Mufti et al. demonstrated an association between the admission neutrophil-to-lymphocyte ratio (NLR) and DCI in patients with aneurysmal $\mathrm{SAH}(\mathrm{aSAH}) .{ }^{5}$ The authors concluded that an admission

ABBREVIATIONS aSAH = aneurysmal SAH; CRP = C-reactive protein; $\mathrm{DCl}$ = delayed cerebral ischemia; $\mathrm{GOS}$ = Glasgow Outcome Scale; NLR = neutrophil-to-lymphocyte ratio; PLR = platelet-to-lymphocyte ratio; ROC = receiver operating characteristic; $\mathrm{SAH}=$ subarachnoid hemorrhage; WFNS = World Federation of Neurosurgical Societies. SUBMITTED October 27, 2021. ACCEPTED December 16, 2021.

INCLUDE WHEN CITING DOI: 10.3171/2021.12.FOCUS21642.

* W.S.B. and P.K.G. contributed equally to this work. R.K.M. and I.A.A. share senior authorship of this work. 
NLR of $\geq 5.9$ was predictive of DCI. ${ }^{5}$ Exploring multiple inflammation markers over time throughout the hospital stay and validating NLR as a predictor in multiple cohorts is essential to providing robust evidence for its prognostic utility. ${ }^{5}$ The primary aims of this study were to assess the reproducibility of NLR to predict DCI and to evaluate how this varies from day to day following the ictus of hemorrhage. Secondary aims included the identification of other inflammatory markers, clinical parameters, and operative parameters as predictors of DCI in aSAH.

\section{Methods \\ Study Population}

A single-center, retrospective study of all patients aged $\geq 18$ years admitted with aSAH between May 2014 and July 2018 was performed at the Leeds General Infirmary. Patients with nonaneurysmal hemorrhage (SAH secondary to perimesencephalic hemorrhage, SAH related to trauma, rupture of an arteriovenous malformation, or other causes) and those aged $<18$ years were excluded.

\section{Clinical Management}

On confirmation of SAH, patients were transferred from their local emergency department to a large, tertiary, neurosurgical center that covers a catchment population of approximately 2 million people, under the care of a specialist neurovascular neurosurgeon and managed in a critical care environment until stabilized (typically $\geq 48$ hours following definitive aneurysm treatment).

Patients were considered to have sustained nonaneurysmal SAH if another clear causal pathology was demonstrated or following both negative CTA and DSA studies. Where a high index of clinical suspicion remained, further images were acquired through repeat CTA or DSA investigations or through MRI of the head and spine.

Unless medically contraindicated, all patients were administered nimodipine prophylaxis at a dose of $60 \mathrm{mg}$ every 4 hours from neurosurgical admission until 21 days postictus. The modality of aneurysm treatment was determined on a case-by-case basis through multidisciplinary discussion between the consultant neurovascular neurosurgeon and consultant interventional neuroradiologist. The majority $(\mathrm{n}=353,87.6 \%)$ of patients were treated by endovascular means, in line with other centers in the United Kingdom. ${ }^{6}$

\section{Data Collection}

Patient demographics, comorbidities, clinical findings, imaging characteristics, incidence of DCI, and interventions were recorded. Outcomes were measured during hospitalization, on discharge, and at 3 months postdischarge. Full blood count differentials were used to identify the absolute neutrophil, lymphocyte, and platelet counts, and these were recorded daily from the date of ictus (day $0 /$ admission) through day 8 postictus or discharge (if discharge occurred $<8$ days after ictus). If $>1$ sample was taken on a specific day, the mean value was used for analysis. Admission values were collected on the day of ictus, such that day 1 values were 1 day postictus and so on. These values were used to calculate the NLR and platelet-to-lymphocyte ratio (PLR). C-reactive protein (CRP) measurements were also recorded as an additional marker of inflammation.

\section{Clinical and Radiological Variables}

The diagnosis of SAH was established by admission CT or by the presence of xanthochromia with lumbar puncture, if the initial CT scan was nondiagnostic. NLR was analyzed using $\mathrm{a} \geq 5.9$ cutoff point, as this value was found to be predictive in previously reported similar studies. ${ }^{5,7}$

DCI was defined as the occurrence of focal neurological impairment (such as hemiparesis, aphasia, apraxia, hemianopia, or neglect) or a decrease of at least two points on the Glasgow Coma Scale that was not apparent immediately after aneurysm occlusion. ${ }^{8}$

\section{Outcome Assessment}

The primary outcome of the study was the incidence of DCI. The Glasgow Outcome Scale (GOS) was used to measure functional outcome at discharge and 3 months postictus. The GOS allows for objective assessment of functional recovery and is scored from 1 to $5(1=$ death to $5=$ low disability). A GOS score $\leq 3$ was considered a poor functional outcome at both assessment time points. A modified Fisher scale grade $\geq 3$ was considered to indicate a dense SAH. Analyses explored which variables might predict DCI.

\section{Statistical Analysis}

Statistical analysis was performed using IBM SPSS Statistics version 24.0.0.0 (IBM Corp.). Descriptive statistics were performed and tabulated on Microsoft Excel version 16.15 to generate summaries of patient baseline characteristics and clinical features, incidence of DCI, and operative features and outcomes. Unless otherwise stated, $\mathrm{p}<0.05$ determined statistical significance.

Logistic regression models were selected via the process outlined below to estimate associations between predictor variables of interest and the primary outcome measure of DCI (yes vs no). The results were expressed as adjusted odds ratios with $95 \%$ confidence intervals. Model selection was performed as follows. 1) The set of predictor variables of interest (see below) were each fitted in a univariate logistic regression model to estimate their (unadjusted) association with incidence of DCI. 2) Those variables that were significantly associated with the outcome of interest at the univariate logistic regression stage were then taken forward as candidate variables for testing in the multiple binary logistic regression stage. 3) A multiple binary logistic regression model was then fitted after controlling for known predictors of DCI, including age, a modified Fisher scale grade of 3 or 4, history of smoking, and history of hypertension, to estimate their (adjusted) association with the incidence of DCI. ${ }^{3,4}$

The initial set of predictor variables of interest were NLR $\geq 5.9$ on admission and for each inpatient day to day 8, PLR $\geq 157$ on admission and for each inpatient day to day $8, C R P \geq 27$ on admission and for each inpatient day to day 8 , age, sex, hypertension, ischemic heart disease, smoking, World Federation of Neurosurgical Societies 
TABLE 1. Patient baseline characteristics and clinical features

\begin{tabular}{|c|c|c|c|c|}
\hline Variable & $\mathrm{DCl}(\mathrm{n}=96)$ & Non-DCl $(n=307)$ & Overall $(n=403)$ & p Value \\
\hline Mean age $\pm S D$, yrs & $54.7 \pm 10.9$ & $56.0 \pm 12.1$ & $55.7 \pm 11.8$ & 0.348 \\
\hline Sex, M/F ratio & $21: 75$ & $94: 213$ & $115: 288$ & 0.098 \\
\hline Hypertensive & $26(40.0)$ & $78(28.9)$ & $104(31.1)$ & 0.086 \\
\hline Status unknown or missing data & 31 & 38 & 69 & \\
\hline History of IHD & $1(1.5)$ & $10(3.9)$ & $11(3.4)$ & 0.342 \\
\hline Status unknown or missing data & 30 & 51 & 21 & \\
\hline History of smoking & $32(47.1)$ & $124(46.2)$ & $156(46.6)$ & 0.827 \\
\hline Status unknown or missing data & 29 & 39 & 68 & \\
\hline \multicolumn{5}{|l|}{ WFNS grade } \\
\hline 1 & $37(38.5)$ & $185(60.2)$ & $222(55.1)$ & $<0.001^{*}$ \\
\hline$\|$ & $24(25.0)$ & $49(16.0)$ & $73(18.2)$ & $0.045^{*}$ \\
\hline III & $2(2.1)$ & $10(3.3)$ & $13(3.2)$ & 0.555 \\
\hline IV & $15(15.6)$ & $24(7.8)$ & $39(9.7)$ & $0.024^{*}$ \\
\hline V & $18(18.8)$ & $38(12.4)$ & $56(13.9)$ & 0.115 \\
\hline \multicolumn{5}{|l|}{ Modified Fisher grade } \\
\hline 1 & $0(0)$ & $13(4.2)$ & $13(3.2)$ & \\
\hline 2 & $0(0)$ & $19(6.2)$ & $19(4.7)$ & \\
\hline 3 & $33(34.4)$ & $146(47.6)$ & $179(44.5)$ & $0.022^{*}$ \\
\hline 4 & $63(65.6)$ & $128(41.7)$ & $191(47.5)$ & $<0.001^{*}$ \\
\hline
\end{tabular}

(WFNS) grade, modified Fisher scale grade, and endovascular treatment.

Receiver operating characteristic (ROC) analysis was performed to evaluate the sensitivity and specificity of NLR $\geq 5.9$ to predict DCI in our cohort, and this was used to identify the corresponding cutoff point, described above, for PLR and CRP.

\section{Results}

\section{Patient Baseline Characteristics and Clinical Features}

A total of 403 patients were included in the final analysis. Patient baseline characteristics and clinical variables are summarized in Table 1 . The mean age \pm SD of the population was $55.7 \pm 11.8$ years, and there were almost twice as many females as males $(\mathrm{M} / \mathrm{F}$ ratio $1: 1.91)$. The majority presented with a WFNS grade of $\mathrm{I}(\mathrm{n}=222$, 55.1\%), and almost all patients had a modified Fisher scale grade of 3 or $4(n=370,91.8 \%)$.

\section{Incidence of $\mathrm{DCl}$}

Ninety-six patients (23.8\%) developed DCI at a median time from ictus of 6 days (IQR 3.25-8). Those patients in whom DCI developed were more likely to have a WFNS grade of IV ( $p=0.024)$, and all had a modified Fisher scale grade of 3 or 4; significantly more patients in the DCI group had a modified Fisher scale grade of 4 than did patients in the non-DCI group $(\mathrm{p}<0.001)$ (Table 1).

\section{Operative and Outcome Data}

SAH treatment and outcomes are summarized in Table
2 . The majority of patients received endovascular treatment for the primary hemorrhage $(\mathrm{n}=353,87.6 \%)$. After treatment, a smaller proportion of patients with DCI were discharged directly to home $(39.6 \%$ vs $68.9 \%$, p < 0.001$)$, more patients with DCI required time in a rehabilitation unit $(41.6 \%$ vs $14.4 \%, \mathrm{p}<0.001)$, and patients with DCI had a longer mean length of stay ( $38.8 \pm 37.4$ days vs 22.3 \pm 27.7 days, $p<0.001$ ).

The overall mortality rate was $11.4 \%(\mathrm{n}=46)$, which was higher in patients with DCI at $14.6 \%(\mathrm{n}=14)$, although this difference did not reach statistical significance $(\mathrm{p}=0.273)$. The proportion of patients with DCI with a GOS score at discharge $\leq 3$ was $41.6 \%(n=48)$ compared with $19.5 \%(n=60)$ of patients without DCI, indicating that the former had poorer function $(\mathrm{p}<0.001)$. This effect remained constant when the GOS score was measured at 3 months postictus. Neither group made a clinically significant functional improvement between assessments (DCI, $\mathrm{n}=30,36.1 \%$ vs non-DCI, $\mathrm{n}=48,16.8 \% ; \mathrm{p}=0.010$ ).

\section{ROC Analysis}

Based on ROC analysis, a value of NLR $\geq 5.9$ yielded a sensitivity of $78.2 \%$ and a specificity of $34.4 \%$ in our cohort. A PLR cutoff $\geq 157$ and a CRP cutoff $\geq 27$ yielded the same sensitivity and specificity as NLR $\geq 5.9$ in our cohort.

\section{Univariate Analysis of Covariates and the Primary Endpoint}

The unadjusted OR estimates, 95\% CIs, and $\mathrm{p}$ values for the univariate logistic regression models of DCI (yes/ 
TABLE 2. Operative and treatment outcome data

\begin{tabular}{|c|c|c|c|c|}
\hline Variable & $\mathrm{DCl}(\mathrm{n}=96)$ & Non-DCl $(n=307)$ & Overall $(n=403)$ & $\mathrm{p}$ Value \\
\hline \multicolumn{5}{|l|}{ Treatment for hemorrhage } \\
\hline MDT involved & $21(56.1)$ & $96(58.5)$ & $117(57.0)$ & 0.777 \\
\hline Status unknown or missing data & 55 & 143 & 198 & \\
\hline Combined & $3(3.1)$ & $3(1)$ & $6(1.4)$ & 0.129 \\
\hline Endovascular & $86(89.5)$ & $267(87)$ & $353(87.6)$ & 0.498 \\
\hline Microsurgical & $7(7.2)$ & $22(7.2)$ & $29(7.2)$ & 0.967 \\
\hline None & $0(0)$ & $15(4.9)$ & $15(3.7)$ & \\
\hline Median day of vasospasm postictus (IQR) & $6(3.25-8)$ & & & \\
\hline \multicolumn{5}{|l|}{ Vasospasm treatment } \\
\hline Medical & $96(100)$ & & & \\
\hline Balloon & $28(29.2)$ & & & \\
\hline Intraarterial nimodipine & $42(43.8)$ & & & \\
\hline Any endovascular treatment & $49(51.0)$ & & & \\
\hline Death & $14(14.6)$ & $32(10.4)$ & $46(11.4)$ & 0.273 \\
\hline Repatriated to local district hospital & $4(4.2)$ & $19(6.2)$ & $23(5.7)$ & 0.448 \\
\hline Discharge home & $38(39.6)$ & $210(68.9)$ & $248(61.5)$ & $<0.001^{*}$ \\
\hline Discharge to rehabilitation unit & $40(41.6)$ & $44(14.4)$ & $84(20.8)$ & $<0.001^{*}$ \\
\hline Mean length of stay $\pm S D$, days & $38.8 \pm 37.4$ & $22.3 \pm 27.7$ & $26.2 \pm 30.7$ & $<0.001^{*}$ \\
\hline GOS score $\leq 3$ at discharge & $40(41.6)$ & $60(19.5)$ & $108(26.8)$ & $<0.001^{*}$ \\
\hline GOS score $\leq 3$ at 3 mos postictus & $30(36.1)$ & $48(16.8)$ & $78(21.1)$ & $0.010^{*}$ \\
\hline Status unknown or missing data & 13 & 22 & 35 & \\
\hline
\end{tabular}

no) versus each of the predictor variables of interest are presented in Table 3 . In our study population, an NLR $\geq$ 5.9 on days 2,3 , and 5 was associated with DCI; a PLR $\geq$ 157 on days 1,2 , and 3 was associated with DCI; and CRP $\geq 27$ on days $2,3,4$, and 5 was associated with DCI.

\section{Multivariate Analysis of Covariates and the Primary Endpoint}

The multivariate predictive model for the endpoint DCI (yes/no) took the form of a multiple binary logistic regression analysis of the endpoint versus each predictor variable of interest after controlling for known predictors of DCI including age, a modified Fisher scale grade of 3 or 4, history of smoking, and history of hypertension.

The final model suggests that after controlling for confounders, the following inflammatory markers remained predictors of DCI (Table 4): day 2 NLR $\geq 5.9$ (OR 2.194, 95\% CI 1.099-4.372; $\mathrm{p}=0.026)$, day 1 PLR $\geq 157$ (OR $2.398,95 \%$ CI $1.072-5.361 ; \mathrm{p}=0.033$ ), day 2 PLR $\geq 157$ (OR 2.676, 95\% CI 1.344-5.329; $\mathrm{p}=0.005$ ), day $3 \mathrm{CRP} \geq$ 27 (OR 2.742, 95\% CI 1.372-5.479; $\mathrm{p}=0.004$ ), day 4 CRP $\geq 27$ (OR 3.268, 95\% CI 1.504-7.101; $\mathrm{p}=0.003)$, and day 5 $\mathrm{CRP} \geq 27$ (OR 3.07, 95 CI 1.221-7.721; $\mathrm{p}=0.017$ ).

\section{Discussion}

The results of the current study demonstrate that DCI remains a common complication after aSAH, especially in patients with high WFNS and modified Fisher scale grades. Patients with DCI had longer hospital stays, required more rehabilitation, and had poorer immediate and medium-term clinical functional outcomes. Our data collection did not extend beyond 3 months, but the literature has suggested that a proportion of these patients will experience poor functional outcomes in the long term as well. ${ }^{9}$ A predictive DCI model based on routinely collected inflammatory biomarkers may allow for early identification of patients at increased risk of DCI and could guide preemptive management to improve their outcomes.

While previous studies have investigated the predictive potential of inflammatory biomarkers at admission only, we have demonstrated the temporal changes in the predictive potential of these markers in patients with aSAH over multiple days after hemorrhage. Our results not only demonstrate the reproducibility of NLR, PLR, and CRP as variables associated with subsequent DCI but also suggest that the predictive potential of these markers is low or absent at admission; rather, they are potentially more predictive when measured on and after day 2 postictus. A recent study exploring the effect of invasive neuromonitoring has demonstrated that the first DCI event was detected earlier, at a mean of 2.2 days postictus. ${ }^{9}$ This earlier detection was associated with a favorable outcome at 12 months. ${ }^{9}$ Our study demonstrates the predictive potential of easily obtained routine biomarkers that are most strongly associated with developing DCI from day 2 onward, representing a new finding, and increases the potential clinical utility of these markers. 
TABLE 3. Univariate binary logistic regression analysis of the endpoint ( $\mathrm{DCl}$ yes/no) versus each predictor variable of interest

\begin{tabular}{|c|c|c|c|}
\hline Variable & OR & $95 \% \mathrm{Cl}$ & $p$ Value \\
\hline Admission NLR $\geq 5.9$ & 1.573 & $0.762-3.248$ & 0.220 \\
\hline Day $1 \mathrm{NLR} \geq 5.9$ & 1.717 & $0.965-3.046$ & 0.066 \\
\hline Day 2 NLR $\geq 5.9$ & 2.463 & $1.401-4.329$ & $0.002^{*}$ \\
\hline Day 3 NLR $\geq 5.9$ & 1.970 & $1.152-3.392$ & $0.013^{*}$ \\
\hline Day 4 NLR $\geq 5.9$ & 1.749 & $0.991-3.087$ & 0.054 \\
\hline Day 5 NLR $\geq 5.9$ & 2.202 & $1.187-4.086$ & $0.012^{*}$ \\
\hline Day 6 NLR $\geq 5.9$ & 1.824 & $0.963-3.453$ & 0.065 \\
\hline Day $7 \mathrm{NLR} \geq 5.9$ & 1.110 & $0.538-2.29$ & 0.778 \\
\hline Day 8 NLR $\geq 5.9$ & 1.253 & $0.525-2.992$ & 0.612 \\
\hline Admission PLR $\geq 157$ & 1.717 & $0.834-3.526$ & 0.142 \\
\hline Day 1 PLR $\geq 157$ & 2.143 & $1.128-4.069$ & $0.020^{*}$ \\
\hline Day 2 PLR $\geq 157$ & 2.613 & $1.503-4.543$ & $0.001^{*}$ \\
\hline Day 3 PLR $\geq 157$ & 1.995 & $1.163-3.422$ & $0.012^{*}$ \\
\hline Day 4 PLR $\geq 157$ & 1.772 & $1.001-3.139$ & 0.050 \\
\hline Day 5 PLR $\geq 157$ & 1.607 & $0.847-3.047$ & 0.147 \\
\hline Day 6 PLR $\geq 157$ & 1.303 & $0.675-2.514$ & 0.431 \\
\hline Day 7 PLR $\geq 157$ & 1.550 & $0.66-3.605$ & 0.309 \\
\hline Day 8 PLR $\geq 157$ & 1.131 & $0.443-2.887$ & 0.798 \\
\hline Admission CRP $\geq 27$ & 1.347 & $0.333-5.442$ & 0.676 \\
\hline Day 1 CRP $\geq 27$ & 1.236 & $0.653-2.338$ & 0.516 \\
\hline Day 2 CRP $\geq 27$ & 1.772 & $1.025-3.061$ & $0.040^{*}$ \\
\hline Day 3 CRP $\geq 27$ & 2.649 & $1.502-4.673$ & $0.001^{*}$ \\
\hline Day 4 CRP $\geq 27$ & 2.839 & $1.517-5.315$ & $0.001^{*}$ \\
\hline Day 5 CRP $\geq 27$ & 1.920 & $1.003-3.764$ & $0.049^{*}$ \\
\hline Day 6 CRP $\geq 27$ & 1.844 & $0.942-3.607$ & 0.074 \\
\hline Day 7 CRP $\geq 27$ & 1.333 & $0.625-2.843$ & 0.457 \\
\hline Day 8 CRP $\geq 27$ & 1.876 & $0.777-4.526$ & 0.162 \\
\hline Age & 0.991 & $0.972-1.010$ & 0.354 \\
\hline Female sex & 1.576 & $0.917-2.708$ & 0.100 \\
\hline Hypertension & 1.632 & $0.931-2.863$ & 0.087 \\
\hline IHD & 0.494 & $0.062-3.924$ & 0.505 \\
\hline Smoking & 1.062 & $0.621-1.815$ & 0.827 \\
\hline Endovascular treatment & 1.742 & $0.75-4.046$ & 0.197 \\
\hline
\end{tabular}

${ }^{*} p<0.05$.

The predictive potential of different variables has been explored in the past. In a prospective clinical study of biomarker predictors, the best admission cutoff values were NLR 14.3 (sensitivity $87.3 \%$ and specificity $48.4 \%$ ) and PLR 193.0 (sensitivity $55.3 \%$ and specificity $78.5 \%$ ) as independent predictors of DCI. ${ }^{10}$ A 2017 review demonstrated a wide variety of biomarkers that had been explored in the literature, including various cytokines, white blood cell differentials, and CRP, as well as physiological systemic inflammatory response syndrome markers, including pyrexia and tachycardia. ${ }^{11}$ While it is clear from our study and the supporting literature that monitoring inflammatory processes can predict DCI, no consensus has been reached as to which parameters, and at what cutoffs, are clinically most useful.
TABLE 4. Multiple binary logistic regression analysis of the endpoint ( $\mathrm{DCl}$ yes/no) versus each predictor variable of interest

\begin{tabular}{cccc}
\hline Variable & OR & $95 \% \mathrm{Cl}$ & p Value \\
\hline Day 2 NLR $\geq 5.9$ & 2.194 & $1.099-4.372$ & 0.026 \\
\hline Day 1 PLR $\geq 157$ & 2.398 & $1.072-5.361$ & 0.033 \\
\hline Day 2 PLR $\geq 157$ & 2.676 & $1.344-5.329$ & 0.005 \\
\hline Day 3 CRP $\geq 27$ & 2.742 & $1.372-5.479$ & 0.004 \\
\hline Day 4 CRP $\geq 27$ & 3.268 & $1.504-7.101$ & 0.003 \\
\hline Day 5 CRP $\geq 27$ & 3.07 & $1.221-7.721$ & 0.017 \\
\hline
\end{tabular}

Model is controlling for known predictors of $\mathrm{DCl}$ including age, a modified Fisher scale grade of 3 or 4 , history of smoking, and history of hypertension.

\section{Limitations}

A weakness of the current study is the retrospective design. A prospective study would benefit from more uniform patient populations and data sets that are less susceptible to bias; however, this would take many years to complete. While our models revealed some interesting associations, they may be underpowered, and any inference should be treated with caution. The estimates of associations between the outcome of interest (DCI) and the predictive variables tested may also be influenced by confounding variables not included in our models, despite our best efforts to include those known to influence the incidence of DCI. For instance, a potential confounder is infection, which was not included in our model. Finally, the majority of patients in this study had modified Fisher scale grades of 3 or 4 , which may limit the generalizability of the results for populations with low blood-load aSAH.

\section{Conclusions}

The evidence that inflammatory processes are involved in the pathophysiology of DCI continues to grow. The identification of optimal biomarkers and understanding of how they contribute to a multiparametric predictive model remain an unmet need. The current study advocates for the inclusion of NLR, PLR, and CRP as biomarker candidates for involvement in future studies, both clinical and mechanistic. We recommend the collection of these markers at admission and to at least day 5 postictus, and for surgeons to consider the early trajectory of these parameters, particularly in patients with aSAH with high WFNS and modified Fisher scale grades.

\section{References}

1. Society of British Neurological Surgeons. National Study of Subarachnoid Haemorrhage; 2006. The Royal College of Surgeons of England. Accessed January 12, 2022. https:// www.sbns.org.uk/index.php/download_file/view/1677/757/

2. Langham J, Reeves BC, Lindsay KW, et al. Variation in outcome after subarachnoid hemorrhage: a study of neurosurgical units in UK and Ireland. Stroke. 2009;40(1):111-118.

3. Budohoski KP, Guilfoyle M, Helmy A, et al. The pathophysiology and treatment of delayed cerebral ischaemia following subarachnoid haemorrhage. J Neurol Neurosurg Psychiatry. 2014;85(12):1343-1353.

4. De Rooij NK, Rinkel GJE, Dankbaar JW, Frijns CJM. Delayed cerebral ischemia after subarachnoid hemorrhage: 
a systematic review of clinical, laboratory, and radiological predictors. Stroke. 2013;44(1):43-54.

5. Al-Mufti F, Amuluru K, Damodara N, et al. Admission neutrophil-lymphocyte ratio predicts delayed cerebral ischemia following aneurysmal subarachnoid hemorrhage. $J$ Neurointerv Surg. 2019;11(11):1135-1140.

6. Anderson IA, Kailaya-Vasan A, Nelson RJ, Tolias CM. Clipping aneurysms improves outcomes for patients undergoing coiling. J Neurosurg. 2019;130(5):1491-1497.

7. Brooks SD, Spears C, Cummings C, et al. Admission neutrophil-lymphocyte ratio predicts 90 day outcome after endovascular stroke therapy. J Neurointerv Surg. 2014;6(8):578-583.

8. Vergouwen MDI, Vermeulen M, van Gijn J, et al. Definition of delayed cerebral ischemia after aneurysmal subarachnoid hemorrhage as an outcome event in clinical trials and observational studies: proposal of a multidisciplinary research group. Stroke. 2010;41(10):2391-2395.

9. Veldeman M, Albanna W, Weiss M, et al. Invasive neuromonitoring with an extended definition of delayed cerebral ischemia is associated with improved outcome after poor-grade subarachnoid hemorrhage. J Neurosurg. 2020;134(5):1527-1534.

10. Tao C, Wang J, Hu X, Ma J, Li H, You C. Clinical value of neutrophil to lymphocyte and platelet to lymphocyte ratio after aneurysmal subarachnoid hemorrhage. Neurocrit Care. 2017;26(3):393-401.

11. Al-Mufti F, Amuluru K, Smith B, et al. Emerging markers of early brain injury and delayed cerebral ischemia in aneurysmal subarachnoid hemorrhage. World Neurosurg. 2017;107:148-159.

\section{Disclosures}

The authors report no conflict of interest concerning the materials or methods used in this study or the findings specified in this paper.

\section{Author Contributions}

Conception and design: Anderson, Akhunbay-Fudge, Chumas, Mathew. Acquisition of data: all authors. Analysis and interpretation of data: Anderson, Bolton, Gharial, Akhunbay-Fudge, Mathew. Drafting the article: all authors. Critically revising the article: all authors. Reviewed submitted version of manuscript: all authors. Approved the final version of the manuscript on behalf of all authors: Anderson. Statistical analysis: Bolton. Administrative/ technical/material support: Bolton, Gharial, Mathew. Study supervision: Anderson, Akhunbay-Fudge, Chumas, Mathew.

\section{Supplemental Information}

\section{Previous Presentations}

The abstract was presented at the British Neurovascular Group Annual Meeting 2020, Stoke-on-Trent, United Kingdom, February 6-7, 2020.

\section{Correspondence}

Ian A. Anderson: Centre for Neurosciences, Leeds Teaching Hospitals NHS Trust, Leeds, United Kingdom. ian.anderson4@ nhs.net. 